\title{
Overproduction of anthocyanin in ginseng hairy roots enhances their antioxidant, antimicrobial, and anti-elastase activities
}

\author{
Sora Jin · Seounggun Bang $\cdot$ Min-A Ahn $\cdot$ Kyubin Lee $\cdot$ Kyunghwan Kim $\cdot$ Tae Kyung Hyun
}

Received: 14 May 2021 / Revised: 28 May 2021 / Accepted: 28 May 2021

(c) Korean Society for Plant Biotechnology

\begin{abstract}
Genetic engineering is a potential approach to improve secondary metabolism in plants. In order to elucidate the effect of production of anthocyanin pigment 1 (PAPI) overexpression on the bioactivity of ginseng, we analyzed its antioxidant, antimicrobial, and anti-elastase activities in this study. Our results showed that $P A P I$ overexpression increased the production of polyphenolic compounds including anthocyanins. The antioxidant, antimicrobial, and anti-elastase activities were stronger in anthocyanin-overproducing ginseng hairy roots (AOX) than in wild ginseng hairy roots. Using a different solvent system $(0,30,70$, and $100 \%$ (v/v) EtOH), we revealed that variations in the contents of the polyphenolic compounds were highly correlated with changes in the antioxidant and antimicrobial activities of AOX. The antioxidant, antimicrobial, and anti-elastase effects of AOX highlight genetic engineering as a powerful approach to enhance the therapeutic properties of plants. Our results show that AOX could potentially have various functional applications in the cosmetic and pharmaceutical industries.
\end{abstract}

Keywords Antioxidant activity, Anthocyanin, Antimicrobial activity, Ginseng hairy root

\section{Introduction}

Anthocyanins are glycosylated polyphenolic compounds synthesized in the cytoplasm and stored in vacuoles (Chanoca

S. Jin $\cdot$ S. Bang $\cdot$ M.-A. Ahn $\cdot$ T. K. Hyun $(\bowtie)$

Department of Industrial Plant Science and Technology, College of Agricultural, Life and Environmental Sciences, Chungbuk

National University, Cheongju 28644, Republic of Korea

e-mail: taekyung7708@chungbuk.ac.kr

K. Lee $\cdot$ K. Kim

School of Biological Sciences, College of Natural Sciences, Chungbuk National University, Cheongju 28644, Republic of Korea et al. 2015). Although anthocyanins are well known as water-soluble flavonoid pigments with colors ranging from orange and red to purple and blue in flowers, seeds, fruits, and vegetation, they are important in attracting pollinators, seed dispersal by promoting fruit consumption, as well as plant protection against biotic and abiotic stresses owing to their antioxidant properties (Liu et al. 2018). There are numerous papers regarding the health benefits of anthocyanins because of their antioxidant, anti-inflammatory, and anticancer effects (Alappat and Alappat 2020), indicating that they are integrally involved interactions between humans and nature. In higher plants, anthocyanin biosynthesis is modulated by the transcriptional complex MYB-bHLH-WD40 (MBW complex) comprising DNA-binding R2R3 MYB transcription factors, basic helix-loop-helix (bHLH) proteins, and WD40 repeat proteins (Xu et al. 2015). Of the three proteins that form the MBW complex, R2R3 MYB transcription factors act as a master regulator of the entire set of anthocyanin biosynthesis genes including chalcone synthase, chalcone isomerase, flavanone 3-hydroxylase, and flavonol synthase (Mehrtens et al. 2005), indicating that the R2R3-MYB transcription factors could be significant in increasing anthocyanin in crops for value-added traits. Ginseng (Panax ginseng C.A. Meyer) is the most widely consumed herbal plant because of its various pharmacological benefits such as neuroprotective, internal secretion adjustment, protective cardiovascular, anti-aging, anti-tumor, and immunomodulatory effects (Choi et al. 2013; Kim 2018). Although ginsenosides, triterpene saponins, are well known as major bioactive ingredients of ginseng, anthocyanins in ginseng berry have been reported to directly inhibit the activity of tyrosinase in $\alpha$-melanocyte-stimulating hormone-stimulated B16F10 cells (Jin et al. 2019). Moreover, the whitening activity of ginseng has been shown to be improved by Production of Anthocyanin Pigment 1 (PAP1, Arabidopsis R2R3-MYB type transcription factor)-dependent anthocyanin accumulation in ginseng hairy roots (Jin and Hyun 2020), 
indicating that the accumulation of anthocyanins in ginseng roots can improve its pharmaceutical properties.

In this study, we examined the antioxidant, antimicrobial, and anti-elastase activities of $P A P 1$-overexpressing ginseng hairy roots to understand the effect of anthocyanins on the pharmaceutical properties of ginseng. This study is expected to garner further interest in the use of anthocyaninoverproducing ginseng roots as a novel functional resource in the cosmetic and pharmaceutical industries.

\section{Materials and methods}

Plant materials and extraction

In a previous study, we generated $P A P 1$-overexpressing ginseng hairy roots using Agrobacterium rhizogenes R1601 (Jin and Hyun 2020). The anthocyanin-overproducing ginseng hairy roots (AOX) and transgenic controls (TC) were cultured on agar-solidified B5 medium at $22{ }^{\circ} \mathrm{C}$ in the dark. Fourweek-old AOX and TC were harvested, ground, and freezedried. The materials were then soaked in different extraction solvents including water, 30, 70, and 100\% (v/v) EtOH for $24 \mathrm{~h}$ and sonicated in an ultrasonic bath. Each solvent extract was evaporated to produce a dried powder extract.

Determination of total phenolic and flavonoid content

In order to determine total phenolic content (TPC), each extract was mixed with $2 \mathrm{~N}$ Folin-Ciocalteu reagent and incubated with $20 \% \mathrm{Na}_{2} \mathrm{CO}_{3}$ for $15 \mathrm{~min}$. The absorbance of the resultant blue colored solution was recorded at a wavelength of $725 \mathrm{~nm}$. The TPC for each extract was calculated using the equation obtained from the standard gallic acid graph and expressed in milligram gallic acid equivalents ( $\mu \mathrm{g} \mathrm{GAE} / \mathrm{mg}$ extract).

Total anthocyanin content (TAC) for each extract was quantified as described by Jin and Hyun (2020). The absorbance was determined at 530 and $657 \mathrm{~nm}$ and the TAC was calculated as (A530 - 0.25*A657) / mg of dry weight.

Chemical-based assays to quantify antioxidant activity

The antioxidant activities of various extracts were determined by monitoring the disappearance of 1,1-dephenyl-2-picrylhydrazyl (DPPH) at $520 \mathrm{~nm}$ and the reducing power assay at $750 \mathrm{~nm}$, as described by Jin et al. (2019). The concentration of sample required to reduce DPPH absorbance by
$50 \%\left(\mathrm{RC}_{50}\right)$ was calculated for each sample.

In order to determine the ferric reducing antioxidant power (the ability to reduce $\mathrm{Fe}^{3+}$ to $\mathrm{Fe}^{2+}$ ), different concentrations of samples were mixed with sodium phosphate buffer $(0.2$ $\mathrm{M}, \mathrm{pH}$ 6.6) and potassium ferricyanide $(1 \%$, w/v). After incubation at $50{ }^{\circ} \mathrm{C}$ for $20 \mathrm{~min}$, the reaction was stopped by adding $10 \%$ trichloroacetic acid. Then, $0.5 \mathrm{ml}$ of reaction mixture was mixed with same volume of distilled water and $0.1 \mathrm{ml}$ of $0.1 \%(\mathrm{w} / \mathrm{v})$ ferric chloride. The absorbance of the sample was measured at $750 \mathrm{~nm}$.

The final assay solution contained $150 \mu \mathrm{l}$ of $0.08 \mu \mathrm{M}$ fluorescein, $25 \mu \mathrm{l}$ of phosphate buffer (blank), Trolox standard $(6.25 \sim 50 \mu \mathrm{M})$, and each extract. After incubation at $37{ }^{\circ} \mathrm{C}$ for $10 \mathrm{~min}$ in the dark, $25 \mu \mathrm{l}$ of $0.12 \mathrm{~g} / \mathrm{ml}$ fresh 2,2'-azobis(isobutyramidine) dihydrochloride was added. A SpectraMax Gemini EM microplate reader was used with fluorescence filters (excitation at $485 \mathrm{~nm}$ and emission at $525 \mathrm{~nm}$ ). The fluorescence of the mixture solution was recorded every minute for $90 \mathrm{~min}$. Area under the curve was calculated for each sample by integrating the relative fluorescence curve. ORAC values were expressed as $\mu \mathrm{M}$ of Trolox equivalents ( $\mu \mathrm{M}$ TE).

Determination of antimicrobial activity

The antimicrobial activity of each extract was tested against eight bacterial species: gram-positive Kocuria rhizophila (KACC 14744), Micrococcus luteus (KACC 14819), Listeria monocytogenes (KACC 19115), and Staphylococcus aureus (KACC 1916) as well as gram-negative Enterobacter cloacae (KACC 11958), Salmonella enteritidis (KACC 12021), Salmonella enterica subsp. enterica (KACC 10769), and Pseudomonas aeruginosa (KACC 2004). The minimum inhibitory concentration (MIC) was determined by an antibacterial assay performed with the two-fold serial dilution method using 96 U-bottom microtiter plates. The lowest concentration showing growth inhibition in comparison with the control was defined as MIC.

Neutrophil elastase inhibition assay

In vitro inhibitory effects of each sample against neutrophil elastase were determined using Neutrophil Elastase Inhibitor Screening Kit (BioVision, Milpitas, CA, USA) according to the manufacturer's instructions.

Statistical analysis

The results were presented as mean \pm standard error (SE) 
of the indicated number of experiments ( $\mathrm{n} \geq 3$ ). One-way analysis of variance (ANOVA) followed by Duncan's multiplerange test was used to determine statistically significant differences between the groups. $P<0.05$ was considered statistically significant.

\section{Results and discussion}

Influence of ethanol concentration on the extraction of phenolic and anthocyanin compounds

Selecting the solvent is one of the most important steps in extraction of bioactive compounds from plant materials. Among pigments, chlorophylls and carotenoids are hydrophobic or nonpolar, whereas anthocyanins are polar molecules (Mattioli et al. 2020; Pérez-Gálvez et al. 2020). Therefore, polar solvents including ethanol and methanol are frequently used for extraction of polyphenolic compounds such as anthocyanins. Considering safety and final potential use in the industry (Wendakoon et al. 2012), we used different concentration of EtOH to compare the extraction efficiency of polyphenolic compounds from AOX and TC. As shown in Figure 1A, the $30 \% \mathrm{EtOH}$ extract of AOX contained the highest TPC of $100.71 \pm 4.17 \mu \mathrm{g}$ GAE$/ \mathrm{mg}$ of extract, whereas the lowest TPC of $27.10 \pm 0.89 \mu \mathrm{g} \mathrm{GAE} / \mathrm{mg}$ of extract was observed in the $100 \%$ EtOH extract of TC. Furthermore, TAC was only detected in AOX samples in the following order: $70 \% \mathrm{EtOH}>30 \% \mathrm{EtOH}>0 \% \mathrm{EtOH}$ (water) $>100 \% \mathrm{EtOH}$ (Fig. 1B). This indicates that a binarysolvent system (EtOH/water) is more effective than a mono-solvent system (water or EtOH) in the extraction of polyphenolic compounds from ginseng hairy roots. It is known that the solvent polarity significantly affects the extraction yield of phytochemicals from plant tissues (Kim 2020). The TPC and TAC changed based on solvent polarity (Fig. 1), indicating that ginseng hairy roots contain diverse polyphenolic compounds with varying polarity.

\section{Antioxidant properties of AOX}

Although reactive oxygen species (ROS) play an important role as intracellular signalling molecules, an imbalance between ROS-generating and ROS-scavenging systems causes oxidative stress, which induces lipid peroxidation, and disrupts DNA, RNA, as well as protein functions (Darbandi et al. 2018). Therefore, antioxidant therapy using free radical scavengers, such as polyphenolic compounds, has been receiving increasing attention as a useful strategy to restore the impaired balance between ROS and antioxidant systems. We hypothesised that that an increase in polyphenolic compounds caused by $P A P 1$ overexpression

A
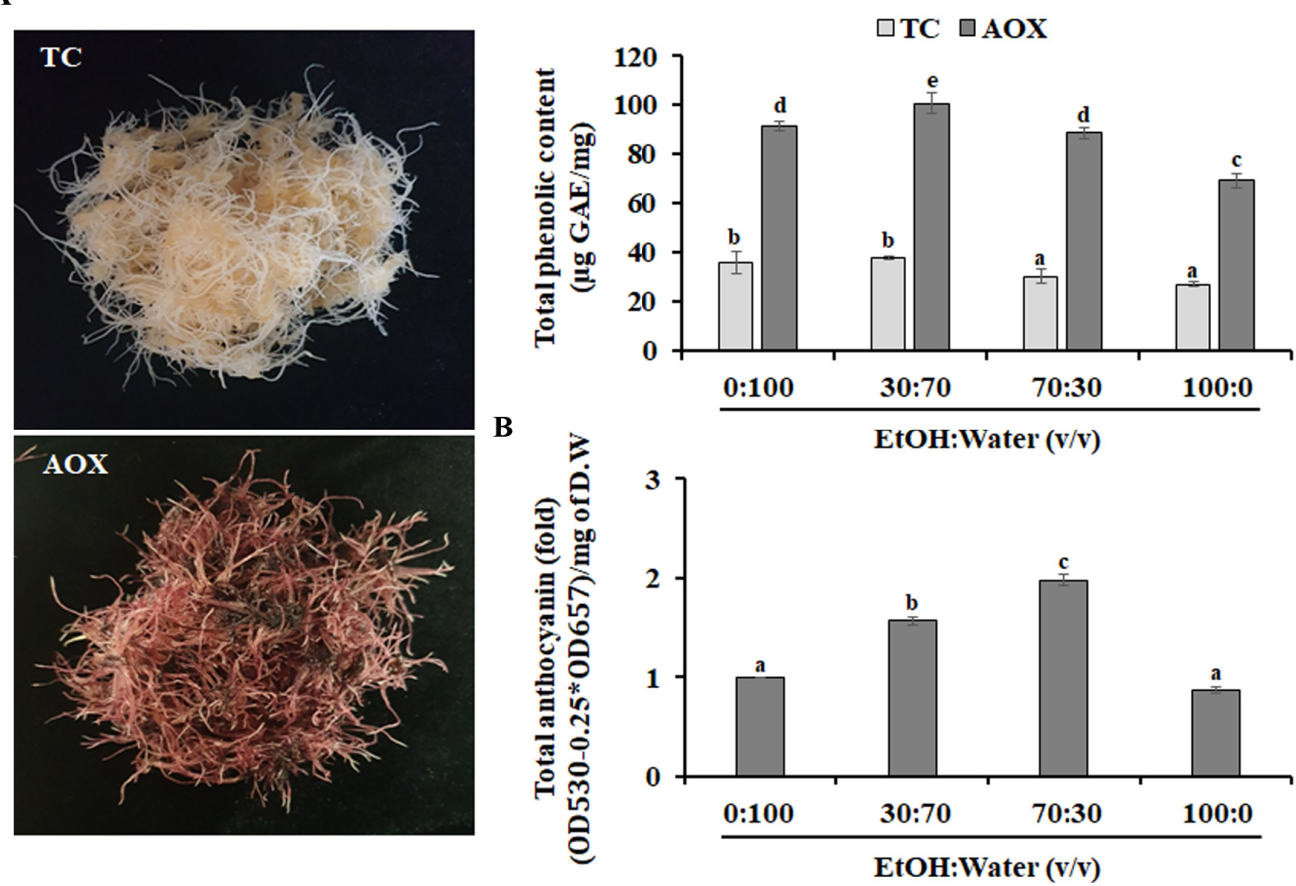

Fig. 1 Effects of PAP1 on total phenol (A) and total anthocyanin contents (B) in ginseng hairy roots. Anthocyanin-overproducing ginseng hairy roots: AOX; Transgenic control ginseng hairy roots: TC. Different letters denote significant differences $(p<0.05$, Duncan's test) 
A

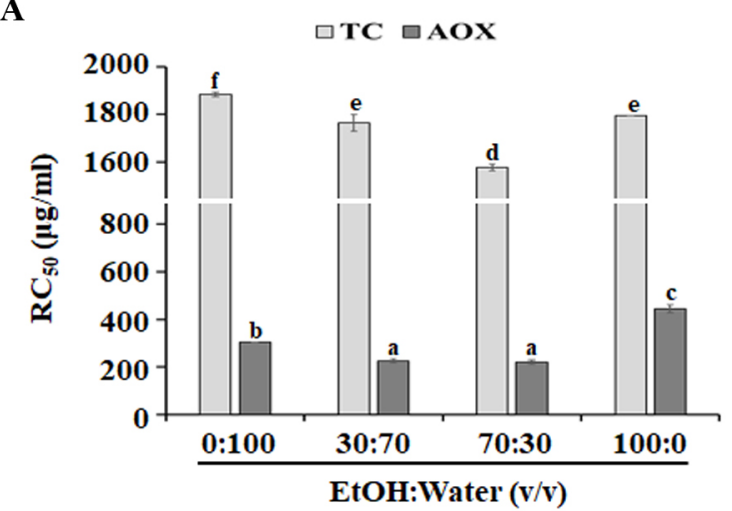

B

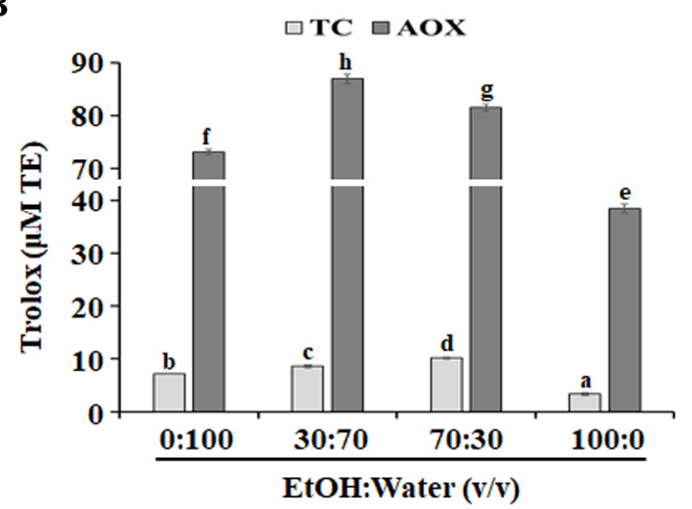

C

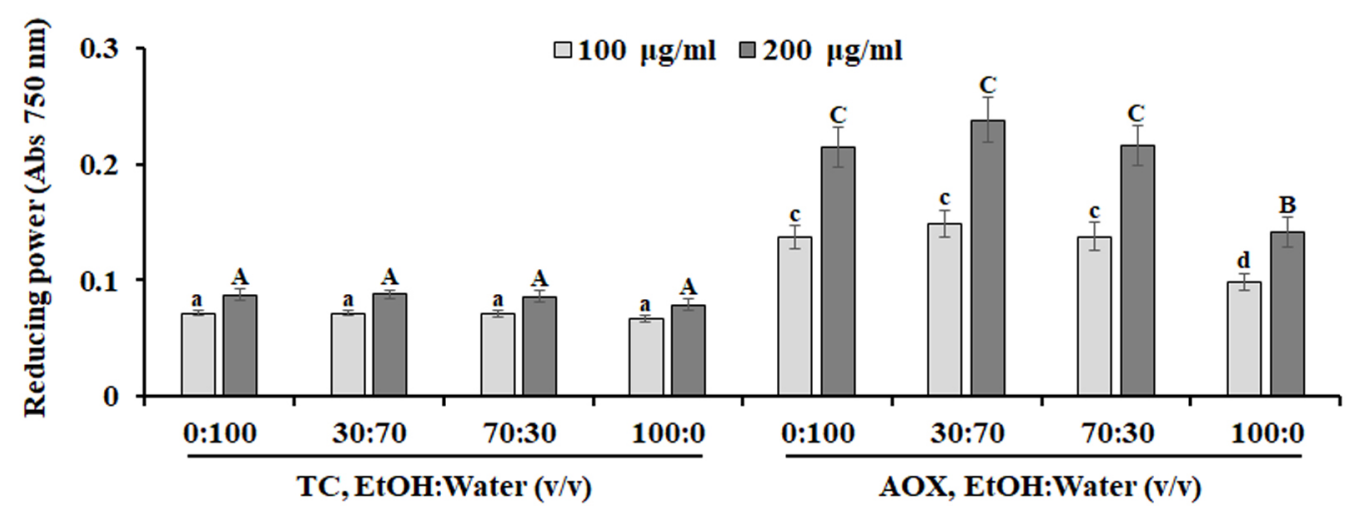

Fig. 2 Antioxidant activity of anthocyanin-overproducing ginseng hairy roots. The antioxidant activity was measured based on DPPH-free radical scavenging activity (A), ORAC assay (B), and reducing power (C). DPPH-radical scavenging activities were expressed as $\mathrm{RC}_{50}$ values $(\mu \mathrm{g} / \mathrm{ml})$. ORAC values for each extract are calculated in $\mu \mathrm{M}$ of Trolox equivalents. Values are averaged from triplicate experiments and represented as mean \pm SE. Different letters denote significant differences $(p<0.05$, Duncan's test). Anthocyanin-overproducing ginseng hairy roots: AOX; Transgenic control ginseng hairy roots: TC

influences the antioxidant properties of ginseng hairy roots. As shown in Figure 2A, AOX extracts exhibited a higher level of DPPH-free radical scavenging activity $\left(\mathrm{RC}_{50}\right.$ between 220.9 and $\left.444.5 \mu \mathrm{g} / \mathrm{ml}\right)$ compared with that exhibited by $\mathrm{TC}\left(\mathrm{RC}_{50}\right.$ between 1577.8 and $\left.1883.9 \mu \mathrm{g} / \mathrm{ml}\right)$. Furthermore, AOX and TC extracts at $40 \mu \mathrm{g} / \mathrm{ml}$ exhibited ORAC values of $38.5 \sim 87.0 \mu \mathrm{M}$ TE and $3.3 \sim 10.2 \mu \mathrm{M}$ TE, respectively (Fig. 2B). Moreover, $200 \mu \mathrm{g} / \mathrm{ml}$ of AOX extracts had OD700 values ranging from 0.14 to 0.24 , which were higher than those of TC (0.08 to 0.09) indicating stronger activity of AOX extracts. AOX extracts that were extracted with 30 and 70\% EtOH exhibited strong DPPHfree radical scavenging activity and high ORAC values, whereas those extracted with $100 \% \mathrm{EtOH}$ exhibited the lowest antioxidant activity (Fig. 2). These results indicate that the polyphenolic constituents are responsible for the antioxidant activities of plants, thereby validating our hypothesis that $P A P 1$ overexpression can improve the antioxidant properties of ginseng hairy roots.
Antimicrobial activity of anthocyanin-overproducing ginseng hairy roots

Despite advancements in modern medicine, infectious diseases remain a major public health problem (Cos et al. 2006). The emergence and dissemination of multidrug-resistant human pathogens have also become a significant public health hazard. The World Health Organization has recognized medicinal plants as a potential source to obtain various antimicrobial agents (Cheesman et al. 2017). Therefore, there has been revived interest in phytochemicals with antimicrobial activities to treat infectious diseases. In order to investigate the antimicrobial activity of ginseng hairy roots, we determined the MIC of each extract using the serial two-fold dilution method (Table 1). Overall, the 30 and $70 \% \mathrm{EtOH} \mathrm{AOX}$ extracts were more effective than the others. The 70\% EtOH AOX extract was most active against Staphylococcus aureus (MIC $=250 \mu \mathrm{g} / \mathrm{ml}$ ) compared to all the bacteria tested. $S$. aureus is the causative agent of multiple infectious human diseases such as bacteremia, skin, prosthetic device, and pulmonary infections (Tong et 
Table 1 Antimicrobial activity of anthocyanin-overproducing ginseng hairy roots

\begin{tabular}{|c|c|c|c|c|c|c|c|c|c|}
\hline \multirow[b]{2}{*}{ Sample } & \multicolumn{9}{|c|}{ MIC $(\mu \mathrm{g} / \mathrm{ml})^{1)}$} \\
\hline & EtOH:Water (v/v) & S. $\mathrm{a}^{2)}$ & M.1 & K.r & L.m & S.e & E.c & S.s & P.a \\
\hline \multirow{4}{*}{$\mathrm{TC}^{3)}$} & $0: 100$ & - & - & 1000 & 1000 & 1000 & 1000 & - & - \\
\hline & $30: 70$ & - & - & 1000 & 1000 & - & 1000 & - & - \\
\hline & $70: 30$ & 1000 & - & 1000 & 1000 & - & 1000 & - & - \\
\hline & 100:0 & - & - & 1000 & - & - & 1000 & - & - \\
\hline \multirow{4}{*}{$\mathrm{AOX}$} & $0: 100$ & - & - & - & 1000 & 500 & 1000 & - & - \\
\hline & $30: 70$ & 1000 & - & 500 & 500 & 500 & 500 & 1000 & - \\
\hline & $70: 30$ & 250 & - & 500 & 500 & 500 & 500 & 1000 & - \\
\hline & $100: 0$ & - & - & 1000 & - & - & 1000 & - & - \\
\hline AMP & & 7.8 & 7.8 & 15.6 & 31.2 & 31.2 & 7.8 & 62.5 & 7.8 \\
\hline
\end{tabular}

${ }^{1)}$ MIC values against bacteria were determined using the two-fold serial dilution method.

${ }^{2)}$ S.a.: Staphylococcus aureus 1916; M.1.: Micrococcus luteus 14819; K.r.: Kocuria rhizophila 14744; L.m.: Listeria monocytogenes 19115; E.c.: Enterobacter cloacae 11958; S.e.: Salmonella enteritidis 12021; S.s.: Salmonellaentrica sub sp.enterica 10769; P.a.: Pseudomonas aeruginosa 2004

${ }^{3)} \mathrm{TC}$ : transgenic control; AOX; anthocyanin-overproduction line; AMP: ampicillin

al. 2015), indicating that AOX is a potential resource to obtain non-antibiotic agents against $S$. aureus. Anthocyanins are antimicrobial agents known to destroy the structure of pathogenic bacteria by inducing cytoplasmic leakage (Ma et al. 2019), indicating that the high level of antimicrobial activity in the 30 and $70 \% \mathrm{EtOH}$ AOX extracts could be because of the presence of high levels of anthocyanins in extracts.

Anti-elastase activity of anthocyanin-overproducing ginseng hairy roots

Wrinkles and loss of skin elasticity are typical phenomena of skin aging, which is caused by the loss of structure of extracellular matrix (ECM) (Trojahn et al. 2015). Degradation of ECM is mainly caused by increasing activity of agingrelated enzymes including elastase (a serine proteinase), which is primarily responsible for the breakdown of elastin in ECM (Pientaweeratch et al. 2016). Therefore, the inhibitors of elastase can be potential cosmetic ingredients to prevent skin aging. As shown in Figure 3, 70\% EtOH AOX extract exhibited the largest inhibitory effect on the elastase activity compared with that of the other extracts. AOX extracted using $70 \% \mathrm{EtOH}(100 \mu \mathrm{g} / \mathrm{ml})$ significantly inhibited elastase activity $(20.5 \pm 0.3 \%)$, whereas AOX extracted using water $(0 \% \mathrm{EtOH})$ showed low inhibitory effects $(8.6 \pm 1.2 \%)$. Similarly, $70 \%$ EtOH TC extract exhibited higher inhibitory activity than other TC extracts. Interestingly, AOX extracts contained higher levels of TPC and TAC than those in TC extracts; however, there was no dramatic difference in the elastase inhibitory activities

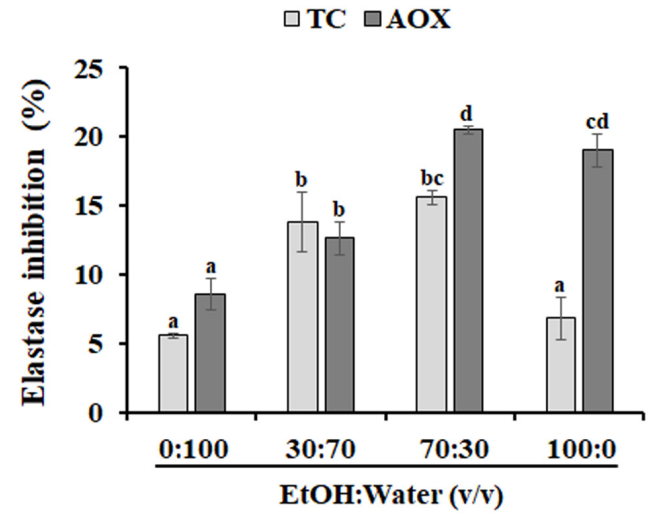

Fig. 3 Anti-elastase activity of anthocyanin-overproducing ginseng hairy roots. Values represent the mean \pm SE from triplicate experiments. Different letters denote significant differences $(p<$ 0.05 , Duncan's test). Anthocyanin-overproducing ginseng hairy roots: AOX; Transgenic control ginseng hairy roots: TC

between the AOX and TC extracts, except the $100 \% \mathrm{EtOH}$ extracts. These results indicate that polyphenolic compounds in ginseng hairy roots are not the major anti-elastase compounds. Ginsenosides have been reported as the main antiaging ingredients in ginseng (Lai et al. 2018). Although some of polyphenolic compounds also act as inhibitors of aging-related enzymes, this finding suggests that the variation in anti-elastase activities between solvent systems could be because of the presence of other active compounds such as ginsenosides.

\section{Conclusion}

In order to determine the biological activities of AOX, we 
analyzed the antioxidant, antimicrobial, and anti-elastase activities of extracts prepared using different solvent systems. We found that AOX extracted using 30 and $70 \% \mathrm{EtOH}$ have strong antioxidant and antimicrobial activities. Although the effects of AOX extracts have been established only in vitro, these results indicate that overproducing anthocyanins through genetic engineering is a promising strategy to improve the pharmaceutical properties of ginseng hairy roots.

\section{Acknowledgements}

This research was supported by Chungbuk National University Korea National University Development Project (2020).

\section{References}

Alappat B, Alappat J (2020) Anthocyanin pigments: Beyond aesthetics. Molecules 25:5500

Chanoca A, Kovinich N, Burkel B, Stecha S, Bohorquez-Restrepo A, Ueda T, Eliceiri KW, Grotewold E, Otegui MS (2015) Anthocyanin vacuolar inclusions form by a microautophagy mechanism. Plant Cell 27:2545-2559

Cheesman MJ, Ilanko A, Blonk B, Cock IE (2017) Developing new antimicrobial therapies: are synergistic combinations of plant extracts/compounds with conventional antibiotics the solution?. Pharmacogn Rev 11:57-72

Choi J, Kim TH, Choi TY, Lee MS (2013) Ginseng for health care: a systematic review of randomized controlled trials in Korean literature. PLoS One. 8:e59978

Cos P, Vlietinck AJ, Berghe DV, Maes L (2006). Anti-infective potential of natural products: how to develop a stronger in vitro 'proof-of-concept'. J Ethnopharmacol 106:290-302

Darbandi M, Darbandi S, Agarwal A, Sengupta P, Durairajanayagam D, Henkel R, Sadeghi MR (2018) Reactive oxygen species and male reproductive hormones. Reprod Biol Endocrinol 16:87

Jin S, Eom SH, Kim JS, Jo IH, Hyun TK (2019) Influence of ripening stages on phytochemical composition and bioavailability of ginseng berry (Panax ginseng C.A. Meyer). J Appl Bot Food Qual 92:130-137

Jin S, Hyun TK (2020) Ectopic expression of production of anthocyanin pigment 1 (PAP1) improves the antioxidant and anti-melanogenic properties of ginseng (Panax ginseng C.A. Meyer) hairy roots. Antioxidants 9:922

Kim JH (2018) Pharmacological and medical applications of Panax ginseng and ginsenosides: a review for use in cardiovascular diseases. J Ginseng Res. 42:264-269

Kim J-S (2020) Effects of solvents with different polarities on the antioxidant activities of the leaves and roots of Allium hookeri. J East Asian Soc Diet Life 30:363-373

Lai M, Zhang H-j, Wang F, Shao Y-1, Yang M-w, Hong F-f, Yang S-1 (2018) Anti-aging effects of ginseng and ginsenosides on the nervous system. Int J Pharmacol 14:1188-1197

Liu Y, Tikunov Y, Schouten RE, Marcelis LFM, Visser RGF, Bovy A (2018) Anthocyanin biosynthesis and degradation mechanisms in Solanaceous vegetables: A review. Front Chem 6:52

Ma Y, Ding U, Fei Y, Liu G, Jang H, Fang J (2019) Antimicrobial activity of anthocyanins and catechins against foodborne pathogens Escherichia coli and Salmonella. Food Control 106:106712

Mattioli R, Francioso A, Mosca L, Silva P (2020) Anthocyanins: a comprehensive review of their chemical properties and health effects on cardiovascular and neurodegenerative diseases. Molecules 25:3809

Mehrtens F, Kranz H, Bednarek P, Weisshaar B (2005) The Arabidopsis transcription factor MYB12 is a flavonol-specific regulator of phenylpropanoid biosynthesis. Plant Physiol 138:1083-1096

Pérez-Gálvez A, Viera I, Roca M(2020) Carotenoids and chlorophylls as antioxidants. Antioxidants 9:505

Pientaweeratch S, Panapisal V, Tansirikongkol A(2016) Antioxidant, anti-collagenase and anti-elastase activities of Phyllanthus emblica, Manilkara zapota and silymarin: an in vitro comparative study for anti-aging applications. Pharm Biol 54:1865-1872

Tong SY, Davis JS, Eichenberger E, Holland TL, Fowler VG Jr (2015) Staphylococcus aureus infections: epidemiology, pathophysiology, clinical manifestations, and management. Clin Microbiol Rev 28:603-661

Trojahn C, Dobos G, Lichterfeld A, Blume-Peytavi U, Kottner J (2015) Characterizing facial skin ageing in humans: disentangling extrinsic from intrinsic biological phenomena. Biomed Res Int 2015:318586

Wendakoon C, Calderon P, Gagnon D (2012) Evaluation of selected medicinal plants extracted in different ethanol concentrations for antibacterial activity against human pathogens. Journal of Medicinally Active Plants 1:60-68

Xu WJ, Dubos C, Lepiniec L (2015) Transcriptional control of flavonoid biosynthesis by MYB-bHLH-WDR complexes. Trends Plant Sci 20:176-185 